\title{
RECEIVED
}

SEP 231996

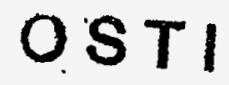

LS - 122(ANL)

M. Yoon and G. Mavrogenes

June 1988

\section{The Focusing Properties of the Positron-Capture Solenoidal Lens}

This note concerns the focusing properties of the positron-capture solenoid. Such a solenoid will be placed before the entrance of the $450 \mathrm{MeV}$ positron linear-accelerator injector in the APS.

The $1.25 \mathrm{~A}, 40 \mathrm{~ns}$ electron beam is accelerated in the $200 \mathrm{MeV}$ electron linac. . This beam is then focused onto a $3 \mathrm{~mm}$ diameter spot at the $7 \mathrm{~mm}$ thick (i.e., two radiation length) tungsten target. By the process of multiple nuclear-scattering the target generates the positron particles. These positrons, in general, can have a large diverging angle. The empirical formula for an angular dependence of the positron yield can be written as:

$$
N(\theta)=N(\theta=0) e^{\left(\frac{-\theta}{\theta_{0}}\right)}
$$

where $\theta_{0} \approx 0.35 \mathrm{rad}(i . e ., \approx 20$ degrees). Here $N(\theta=0)$ is the forward yield.

In order to capture these rapidly diverging positrons, a relatively strong fo- . cusing lens is placed close to the converter. In this case a magnetic solenoidal lens has an advantage over the usual quadrupole lens because of its larger phasespace acceptance. In particular, the solenoidal lens is noted for its capability of controlling the spin direction of polarized ions. This type of lens, however, has the disadvantage that for a given focal length it requires much more power than

the quadrupole lens. When a solenoid requires high power, it is imperative to pulse it in order to reduce the time-averaged power. 


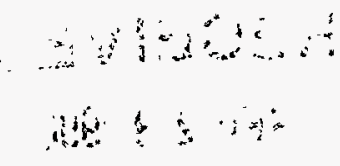

In investigating the particle motion through the solenoidal lens, one usually assumes a uniform field distribution inside the solenoid. The radial components of the field are assumed to exist only at the entrance and at the exit of the lens. This approximation holds well when one treats the paraxial ray only. However, this is not the case for the positron-capture solenoid described here (i.e., positrons have large diverging angle). Therefore, one should study the focusing properties of the solenoid arising from the nonlinearity of the magnetic field.

We shall first write down the expression for the magnetic field distribution on the axis for the solenoidal lens. From this expression we derive the expressions for the first- and second-order field distributions at any point in the region. We then calculate and compare the focal powers at various distances off the axis. Further, we calculate and include the third- and fourth-order components of the fields and compare them with the previous results. Finally, we briefly consider the particle trajectories through these fields and present the result in terms of the particle motion in phase space.

\section{Magnetic field distribution of a double-layer solenoid}

The magnetic fields of a single-layer solenoidal lens can be easily calculated by assuming that the solenoid is a series of closely packed circular current loops. This assumption remains valid as long as the pitch of the solenoid is small compared with the total length of the solenoid. Then the vector potential at any point in the region can be expressed by the following equation:

$$
A_{\phi}=\frac{\mu_{0} I}{\pi k}\left(\frac{a}{\rho}\right)^{1 / 2}\left[\left(1-\frac{k^{2}}{2}\right) K(k)-E(k)\right]
$$




\section{DISCLAIMER}

This report was prepared as an account of work sponsored by an agency of the United States Government. Neither the United States Government nor any agency thereof, nor any of their employees, makes any warranty, express or implied, or assumes any legal liability or responsibility for the accuracy, completeness, or usefulness of any information, apparatus, product, or process disclosed, or represents that its use would not infringe privately owned rights. Reference herein to any specific commercial product, process, or service by trade name, trademark, manufacturer, or otherwise does not necessarily constitute or imply its endorsement, recommendation, or favoring by the United States Government or any agency thereof. The views and opinions of authors expressed herein do not necessarily state or reflect those of the United States Government or any agency thereof. 


\section{DISCLAIMER}

Portions of this document may be illegible in electronic image products. Images are produced from the best available original document. 
where

$$
k^{2}=\frac{4 a \rho}{(a+\rho)^{2}+z^{2}}
$$

In the above equation for $A_{\phi}, \mu_{0}$ is the permeability in free space, $I$ is the total current in ampere-turns, $a$ is the radius in meters, and $K$ and $E$ are the complete elliptic integrals of the first- and the second-kind, respectively. The magnetic fields then can be determined from $\vec{B}=\nabla \times \vec{A}$ :

$$
B_{\rho}=-\frac{\partial A_{\phi}}{\partial z}, \quad B_{z}=\frac{1}{\rho} \frac{\partial}{\partial \rho}\left(\rho A_{\phi}\right)
$$

Carrying out the differentiations, we finally get

$$
\begin{aligned}
& B_{\rho}=\frac{\mu_{0} I}{2 \pi} \frac{z}{\rho\left[(a+\rho)^{2}+z^{2}\right]^{1 / 2}}\left[-K(k)+\frac{a^{2}+\rho^{2}+z^{2}}{(a-\rho)^{2}+z^{2}} E(k)\right] \\
& B_{z}=\frac{\mu_{0} I}{2 \pi} \frac{1}{\left[(a+\rho)^{2}+z^{2}\right]^{1 / 2}}\left[K(k)+\frac{a^{2}-\rho^{2}-z^{2}}{(a-\rho)^{2}+z^{2}} E(k)\right]
\end{aligned}
$$

The calculation of the field for a double-layer solenoid involves complicated integrations and differentiations of the elliptic integrals. However, one can obtain an accurate field distribution by using a field property for a rotationally symmetrical geometry. That is, the field in the region of interest can be evaluated if one knows the field distribution on the axis. The field off the axis can then be obtained from the Taylor expansion of the field on the axis:

$$
\begin{aligned}
& B_{z}(\rho, z)=B_{z}(0, z)-\frac{\rho^{2}}{4}\left(\frac{\partial^{2} B_{z}(0, z)}{\partial z^{2}}\right)+\frac{\rho^{4}}{64}\left(\frac{\partial^{4} B_{z}(0, z)}{\partial z^{4}}\right)-\cdots \\
& B_{\rho}(\rho, z)=-\frac{\rho}{2}\left(\frac{\partial B_{z}(0, z)}{\partial z}\right)+\frac{\rho^{3}}{16}\left(\frac{\partial^{3} B_{z}(0, z)}{\partial z^{3}}\right)-\frac{\rho^{5}}{384}\left(\frac{\partial^{5} B_{z}(0, z)}{\partial z^{5}}\right)+\cdots
\end{aligned}
$$

For a double-layer solenoid, the calculation of the field along the axis is straight- 
forward, and the result is given by

$$
\begin{aligned}
B(0, z) & =\frac{\mu_{0} I N}{2(b-a) L}\left[\left(z-\frac{L}{2}\right) \ln \frac{b+\sqrt{b^{2}+\left(z+\frac{L}{2}\right)^{2}}}{a+\sqrt{a^{2}+\left(z+\frac{L}{2}\right)^{2}}}\right. \\
& \left.-\left(z-\frac{L}{2}\right) \ln \frac{b+\sqrt{b^{2}+\left(z-\frac{L}{2}\right)^{2}}}{a+\sqrt{a^{2}+\left(z-\frac{L}{2}\right)^{2}}}\right]
\end{aligned}
$$

where $a$ and $b$ are the inner and the outer radii in meters, $L$ is the total length in meters, and $N$ is the total number of windings on both layers (i.e., $N=N_{1}+N_{2}$ ) of the solenoid. From this expression, one can obtain the first- and secondderivatives, etc. The results are:

$$
\begin{aligned}
\frac{\partial B_{z}}{\partial z}= & C_{a}\left(\ln B_{+}-\ln A_{+}\right) \\
& +C_{a}\left(z+\frac{L}{2}\right)^{2}\left[\frac{1}{B_{+} \beta_{+}}-\frac{1}{A_{+} \alpha_{+}}\right] \\
& -C_{a}\left(\ln B_{-}-\ln A_{-}\right) \\
& -C_{a}\left(z-\frac{L}{2}\right)^{2}\left[\frac{1}{B_{-} \beta_{-}}-\frac{1}{A_{-} \alpha_{-}}\right]
\end{aligned}
$$

and

$$
\begin{aligned}
\frac{\partial^{2} B_{z}}{\partial z^{2}} & =3 C_{a}\left(z+\frac{L}{2}\right)\left[\frac{1}{B_{+} \beta_{+}}-\frac{1}{A_{+} \alpha_{+}}\right] \\
& -3 C_{a}\left(z-\frac{L}{2}\right)\left[\frac{1}{B_{-} \beta_{-}}-\frac{1}{A_{-} \alpha_{-}}\right] \\
& -\frac{C_{a}\left(z+\frac{L}{2}\right)^{3}}{B_{+} \beta_{+}^{2}}\left[\frac{1}{B_{+}}+\frac{1}{\beta_{+}}\right] \\
& +\frac{C_{a}\left(z+\frac{L}{2}\right)^{3}}{A_{+} \alpha_{+}^{2}}\left[\frac{1}{A_{+}}+\frac{1}{\alpha_{+}}\right] \\
& +\frac{C_{a}\left(z-\frac{L}{2}\right)^{3}}{B_{-} \beta_{-}^{2}}\left[\frac{1}{B_{-}}+\frac{1}{\beta_{-}}\right] \\
& -\frac{C_{a}\left(z-\frac{L}{2}\right)^{3}}{A_{-} \alpha_{-}^{2}}\left[\frac{1}{A_{-}}+\frac{1}{\alpha_{-}}\right]
\end{aligned}
$$


where

$$
\begin{aligned}
& C_{a}=\frac{\mu_{0} I N}{2(b-a) L} \\
& A_{+}=a+\sqrt{a^{2}+(z+L / 2)^{2}} \\
& \alpha_{+}=\sqrt{a^{2}+(z+L / 2)^{2}} \\
& B_{+}=b+\sqrt{b^{2}+(z+L / 2)^{2}} \\
& \beta_{+}=\sqrt{b^{2}+(z+L / 2)^{2}} \\
& A_{-}=a+\sqrt{a^{2}+(z-L / 2)^{2}} \\
& \alpha_{-}=\sqrt{a^{2}+(z-L / 2)^{2}} \\
& B_{-}=b+\sqrt{b^{2}+(z-L / 2)^{2}} \\
& \beta_{-}=\sqrt{b^{2}+(z-L / 2)^{2}}
\end{aligned}
$$

One can go on further to obtain higher-order terms, but the number of terms increases rapidly and subsequently the calculation becomes more and more complicated. At this point, therefore, it is expedient to resort to the numerical method to calculate higher-order terms. This can be done by using the numerical derivatives by polynomial interpolation. A computer program was written to calculate the general field distribution of a double-layer solenoidal lens up to any given order in the field expansion. The result obtained by this numerical method was compared with the above analytical expression up to the second order and was found to be the same. From here on, when we say the higher-order magnetic field, it will mean that the magnetic field expansion includes the terms up to the fourth order. 


\section{Motion of the positrons through the solenoidal lens}

Some parameters for the APS positron-capture solenoidal lens are listed in the following:

$$
L=4.6 \mathrm{~cm}, a=1.25 \mathrm{~cm}, b=2.25 \mathrm{~cm}, N=16 \text { turns, } I \approx 5000 \mathrm{~A}
$$

These parameters are based on the DESY design. We assume that the solenoid is placed at $5 \mathrm{~mm}$ distance from the target. With these parameters, we show in Fig. 1 the variations of $B_{z}$ on the axis and off the axis. The off-axis field distribution was obtained from the fourth-order expansion of the field on the axis (i.e., Eq. (6)). Fig. 1 indicates that the nonlinear effect is not as significant as we conjectured in the first place. More explicit comparison between the linear and the nonlinear effect can be made by considering the focusing power of the lens at various radii.

The focusing power of the solenoid can be easily derived from the radial equation of motion in cylindrical coordinates $(\rho, \phi, z)$. It is given by:

$$
\gamma M_{0} \frac{d^{2} \rho}{d t^{2}}=-e B_{z} \rho \frac{d \phi}{d t}+\gamma M_{0} \rho\left(\frac{d \phi}{d t}\right)^{2}
$$

where $M_{0}$ is the rest mass of the particle, $\gamma$ is the usual relativistic factor, and $\phi$ is the azimuthal angle. By using the Busch's theorem assuming the initial flux linked on the trajectory is zero (i.e., $\Phi_{0}=0$ ),

$$
\frac{d \phi}{d t}=\frac{e}{2 \pi \gamma \rho^{2}}\left(\Phi-\Phi_{0}\right)=\frac{e}{M_{0} \gamma} \frac{B_{z}}{2}
$$

and changing the independent variable from $t$ to $z$, which is the distance along the axis of the lens, and integrating the equation with respect to $z$, one can finally 
arrive at the expression for the focusing power of the axial magnetic lens: .

$$
\frac{1}{f}=\left(\frac{e}{2 M_{0} \gamma \beta c}\right)^{2} \int_{-\infty}^{+\infty} B_{z}^{2} d z
$$

where $\beta=v / c$ and $c$ is the speed of light. In the case of the APS positrons generated by the target, the central energy is assumed to be $8 \mathrm{MeV}$. This corresponds to $\gamma=16.6556, \beta=0.998196$. With these values, Fig. 2 summarizes the focusing power at different radii.

By using the fields obtained above, one can trace a number of positrons from the positron target to the further downstream. In the following, we present some preliminary results.

The radial and angular, as well as the energy, distribution of the positrons generated by the converter can be obtained by the Monte-Carlo calculation of the 6. electromagnetic shower. However, since we are not concerned with the detailed distribution of the positrons (i.e., beam brightness), we assume that the particles are distributed uniformly in radius and angle. This assumption is permissible if one is interested in obtaining the acceptance of the lens. The energy spread is not included in our preliminary calculation, which will be taken into account later in a more detailed paper: Further, the space-charge effect was not taken into account in the calculation since the positrons are already relativistic (i.e., space-charge force decreases as $\left.1 / \gamma^{2}\right)$.

The positron-capture solenoid acts like a quarter-wave transformer. By this we mean that the role of the solenoid merely rotates the initial phase-space diagram by 90 degrees. That is, large angular divergence and small radial spread of 
the initial positrons are transformed into small divergence and large radial spread of the final positrons. That this really holds in our case is demonstrated in Fig. 3 (a) and (b). In Fig. 3, (a) depicts the transverse phase-space diagram at the position of the target, and (b) describes the transverse phase-space diagram further downstream after the solenoid (i.e., $z=10 \mathrm{~cm}$ ) where the field of the solenoidal lens is almost zero. In this figure, we take about 80 uniformly distributed extreme particles at the position of the target. The chosen initial conditions are such that $x \approx \pm 1.5 \mathrm{~mm}, x^{\prime} \approx \pm 210 \mathrm{mrad}$. From the figure, one can clearly see the focusing property of the solenoid so that the initial large divergence $(1.5 \mathrm{~mm}$ $\times 210 \mathrm{mrad})$ is converted to $(7.5 \mathrm{~mm} \times 42 \mathrm{mrad})$. The slight filamentation (i.e., distortion) of the phase-space comes from the nonlinear magnetic field that we discussed before. This phenomenon is well known and would not appear in the first-order theory.

A further study indicates that the acceptance of the solenoid is about $360 \mathrm{~mm}$ mrad in both transverse planes when the current is optimized to 5000 amperes per turn (i.e. $B_{0}=1.81 \mathrm{~T}$ at the center). The overall acceptance of the positron transport system, however, is reduced when we take into account the first two sections of the linear accelerator where long solenoids are placed around the accelerating waveguides. The description of the results of this study is the subject of the next note. 


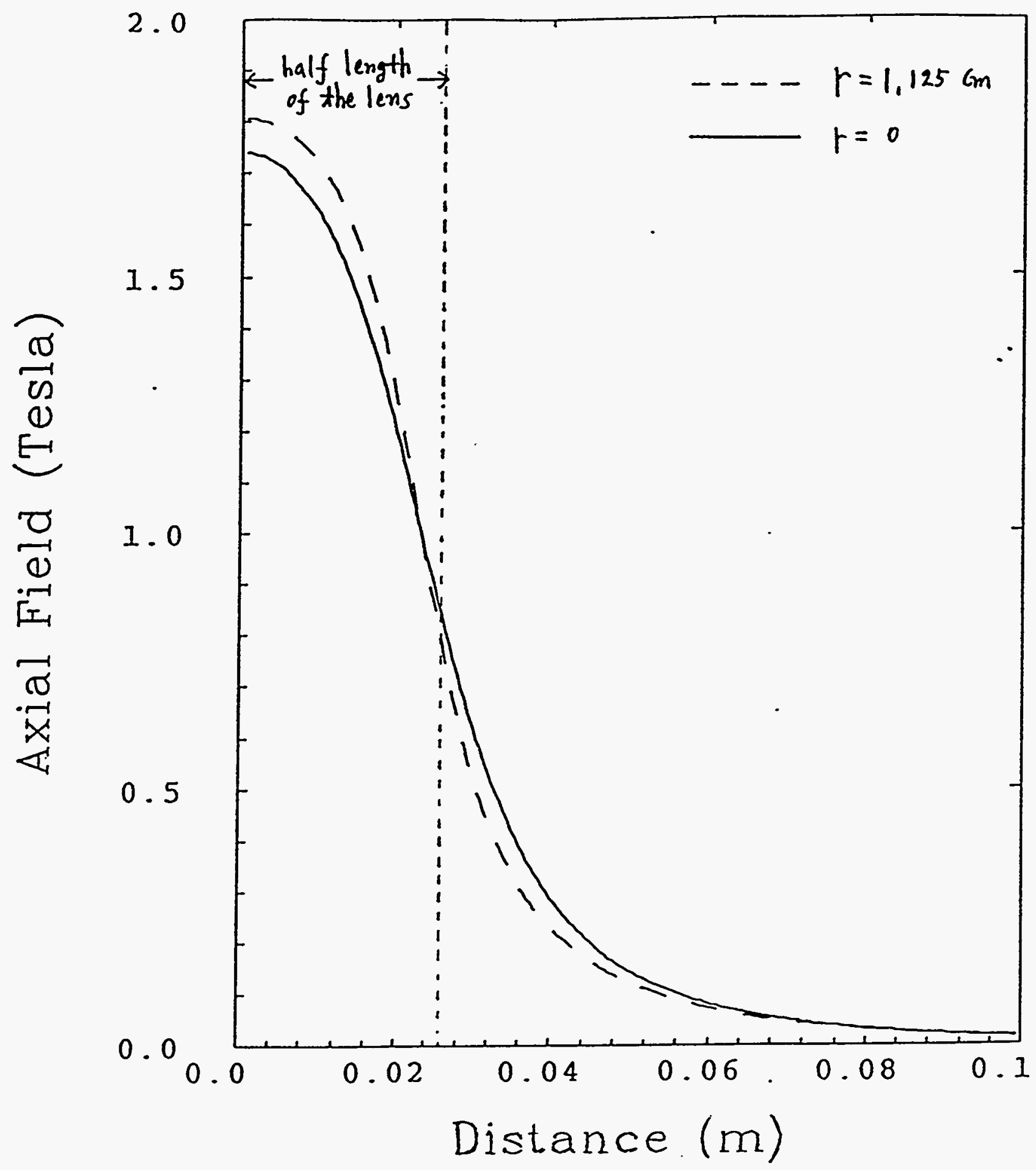

Fig. 1 Axial magnetic fields of the positron-capture solenoid. The solid curve represents the axial magnetic field at $\mathrm{r}=0 \mathrm{~cm}$. The dotted curve descrives the axial magnetic field at $r=1.125 \mathrm{~cm}$. 


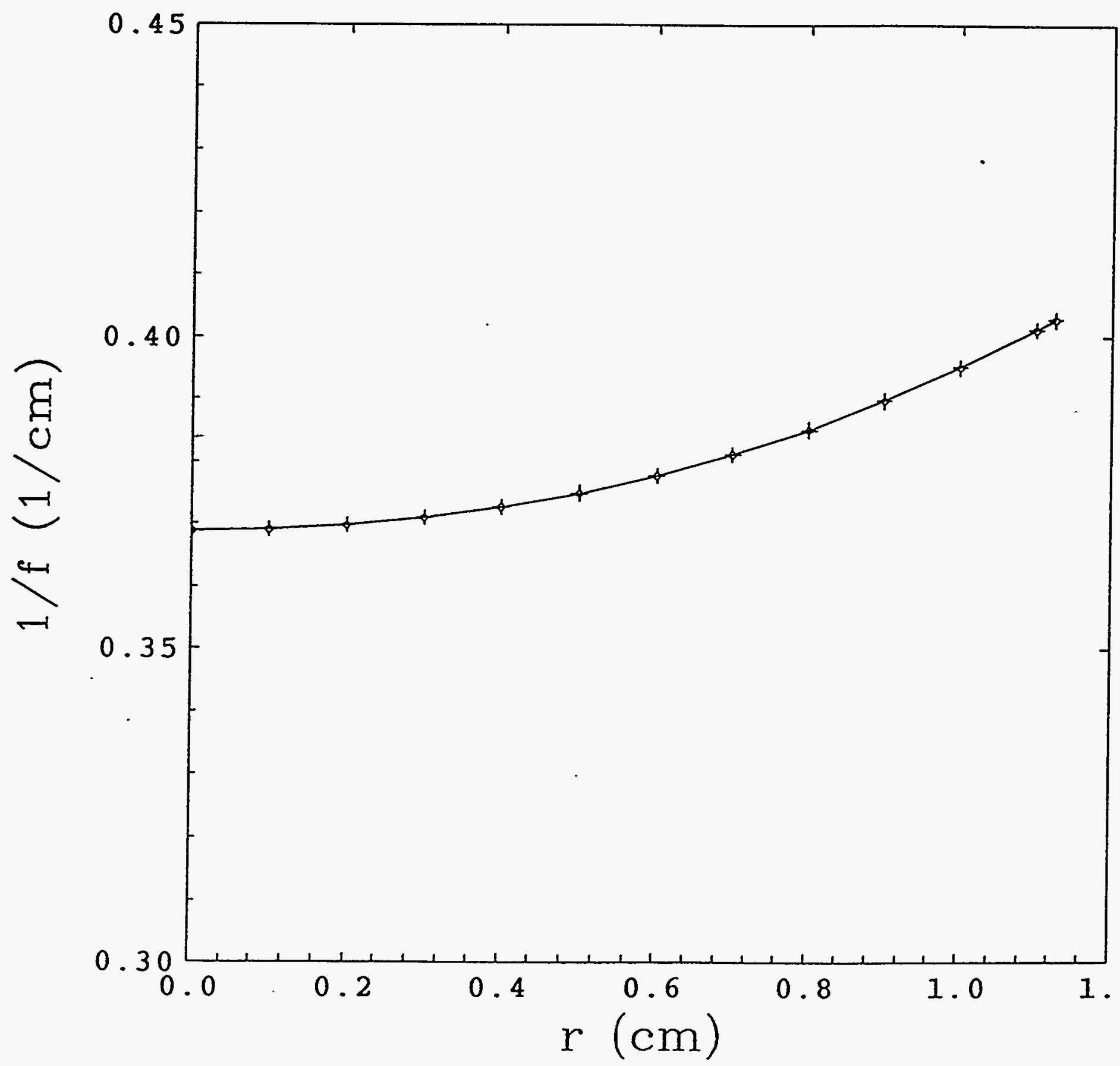

Fig. 2 The focusing power of the position-capture solenoid as a function of radius. 


\section{Transverse Phase Space}

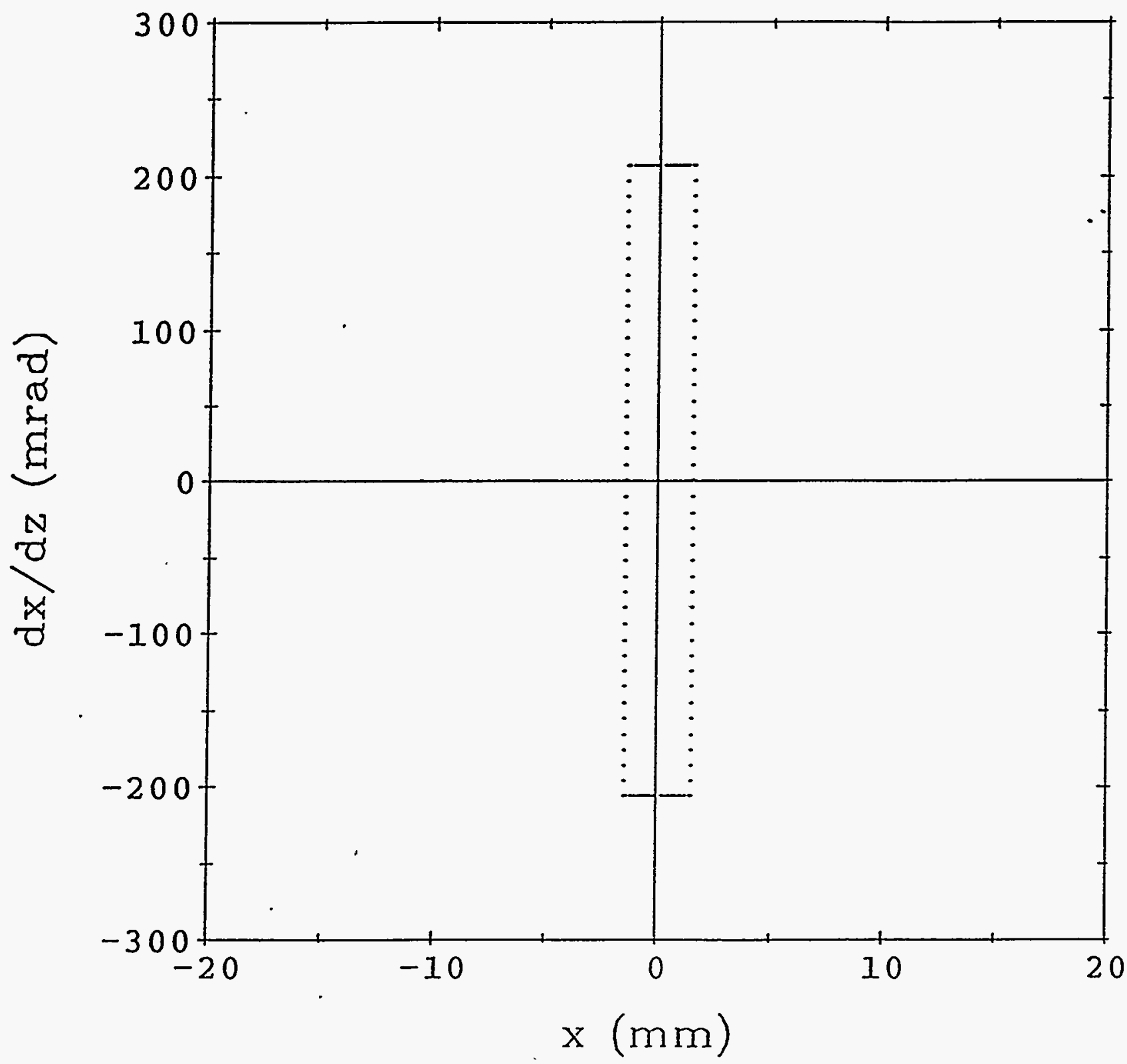

Fig. 3(a) The transverse phase space of particles at the position of target. 


\section{Transverse Phase Space}

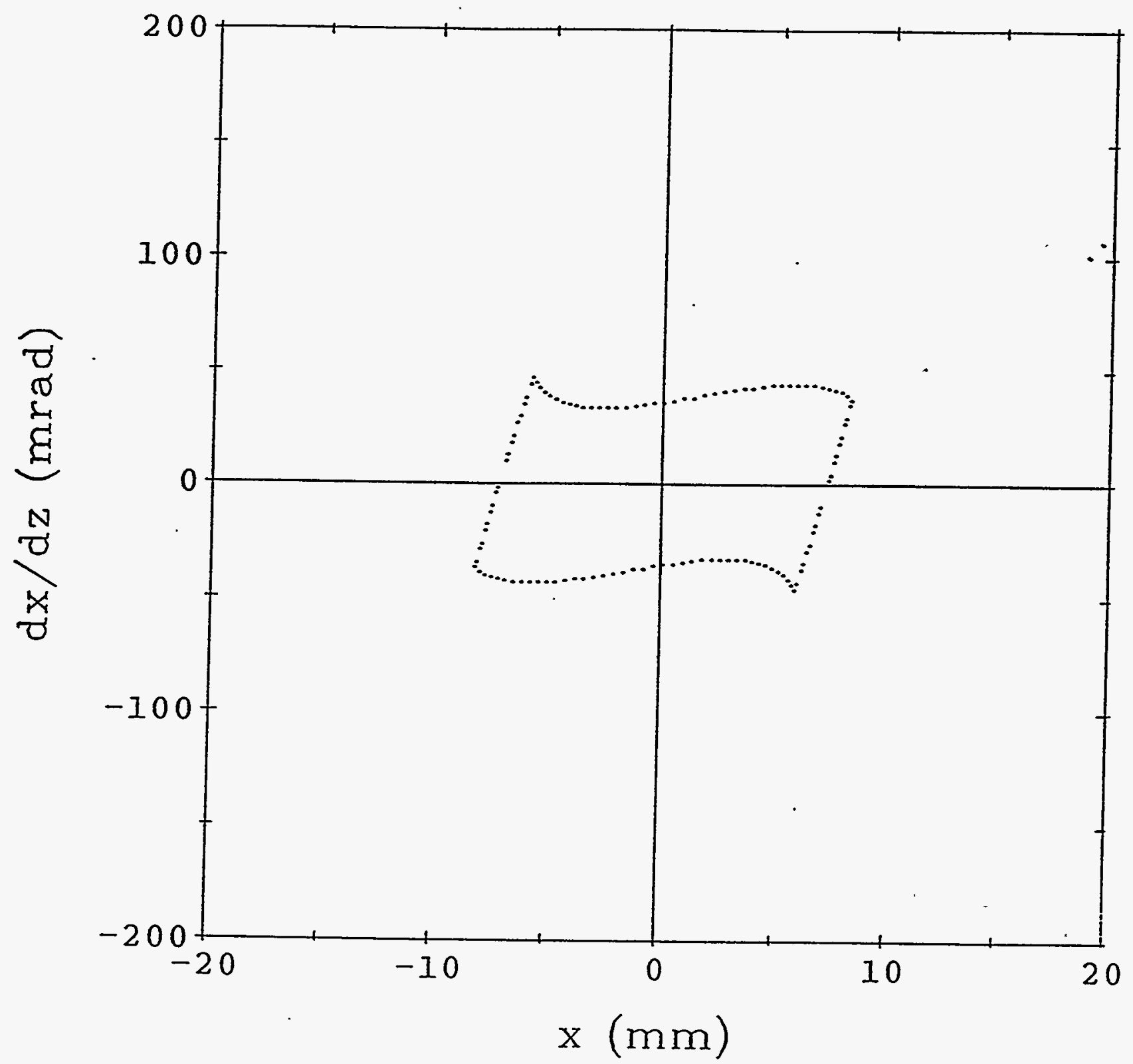

Fig. 3(b) The transverse phase space of particles further downstream $(z=10 \mathrm{~cm})$ after the solenoid. Uniform distribution in angle and displacement is assumed. The initial energy spread is not included in these figures. 